\title{
Synthesis of Poly(phenylene polysulfide) Networks from Elemental Sulfur and $p$-Diiodobenzene for Stretchable, Healable, and Reprocessable Infrared Optical Applications
}

Ji Mok Lee, ${ }^{+, \dagger}$ Guk Yun Noh, ${ }^{+}$Byoung Gak Kim, ${ }^{+}$Youngjae Yoo, ${ }^{+}$Woo Jin Choi, ${ }^{\S}$ Dong-Gyun Kim, ${ }^{*,+}$ Ho Gyu Yoon, ${ }^{*,+}$ and Yong Seok Kim ${ }^{*,+}$

${ }^{+}$Advanced Materials Division, Korea Research Institute of Chemical Technology, 141 Gajeong-ro, Yuseong-gu, Daejeon 34114, Republic of Korea

${ }^{\ddagger}$ Department of Materials Science and Engineering, Korea University, Seoul 02841, Republic of Korea

${ }^{\circledR}$ Chemical Materials Solutions Center, Korea Research Institute of Chemical Technology, 141 Gajeong-ro, Yuseong-gu, Daejeon 34114, Republic of Korea

*Corresponding author: D.-G. Kim (E-mail:dgkim@krict.re.kr)

H.G.Yoon (E-mail:hgyoon@korea.ac.kr)

Y.S.Kim (E-mail: yongskim@krict.re.kr)

$\underline{\text { Table of Contents }}$

Experimental Section

2

Synthesis of Poly(phenylene polysulfide) Networks (PSNs) 6

Structural Analysis of PSNs

Mechanical Characterization of PSN Films

Optical Properties of PSN Films

References

for

Supporting

Information 


\section{Experimental Section}

\section{Materials}

Elemental sulfur (ES, $\geq 99.5 \%$ ), $p$-diiodobenzene (DIB, 99\%), and toluene ( $\geq 99.5 \%$ ), all from Sigma-Aldrich, were used as received. Silicone oil (KF-54) was purchased from Shin-Etsu. All other reagents and solvents were used as received from standard vendors.

\section{Synthesis of poly(phenylene polysulfide) networks (PSNs)}

Poly(phenylene polysulfide) networks are designated as PSN\#s, where \# indicates the average weight percentage ( $\mathrm{wt} \%)$ of sulfur in the polymer. The following procedure was used for the synthesis of PSN76 in Table 1. ES (2.3 g, $70 \mathrm{mmol})$, DIB ( $3.3 \mathrm{~g}, 10 \mathrm{mmol})$, and silicone oil $(5.0 \mathrm{~mL})$ were placed into a $100 \mathrm{~mL}$ 2-neck round-bottomed flask with a magnetic stirring bar. The reaction flask sealed with stopper was transferred to a thermostatted oil bath at $60^{\circ} \mathrm{C}$. After stirred for $30 \mathrm{~min}$ under a nitrogen $\left(\mathrm{N}_{2}\right)$ flow, the monomers were melted by heating at $130^{\circ} \mathrm{C}$ for $30 \mathrm{~min}$. Finally, the temperature was raised to $230{ }^{\circ} \mathrm{C}$ and the polymerization was conducted for $18 \mathrm{~h}$. The $\mathrm{N}_{2}$ gas was continuously flowed from one neck to the other to remove the generated $I_{2}$ during the polymerization. The $\mathrm{N}_{2}$ outlet was connected to a cold trap for the $\mathrm{I}_{2}$ recovery. After cooling to room temperature, the resulting polymer was washed several times with acetone, and subsequently dried under vacuum at $70^{\circ} \mathrm{C}$ overnight to yield a brownish solid (2.1 g, 63\%). Other PSNs were prepared using the same procedure, varying the ratio of the DIB and ES monomer charges; molar feed ratios ([DIB]:[ES]) of 1:1 to 1:6 were used for PSNs with sulfur content of $33 \mathrm{wt} \%$ to $72 \mathrm{wt} \%$ (Table 1). Average sulfur ranks $(N)$ in the PSNs (i.e., average number of sulfur atoms in the polysulfide linkages $)$ were calculated by $N=\left(W_{\mathrm{s}} / M_{\mathrm{s}}\right) /\left[\left(1-W_{\mathrm{s}}\right) / M_{\mathrm{ar}}\right]$, where $W_{\mathrm{s}}$ is the weight fraction of sulfur in the PSNs, and $M_{\mathrm{s}}\left(32 \mathrm{~g} \mathrm{~mol}^{-1}\right)$ and $M_{\mathrm{ar}}\left(76 \mathrm{~g} \mathrm{~mol}^{-1}\right)$ are molecular weights of sulfur atom and aromatic moiety of DIB $\left(\mathrm{C}_{6} \mathrm{H}_{4}\right.$ without iodine $)$, respectively. For this calculation, we assumed that the random branching reactions on the $\mathrm{C}-\mathrm{H}$ bonds of the aromatic ring to form cross-linked networks are negligible (i.e., the linear poly(phenylene polysulfide) chains are dominant in the PSNs), because it is difficult to detect and quantify the minor, random cross-linking sites in the PSNs.

\section{Preparation of PSN films}


PSN films were prepared by placing the polymers into a mold $(40 \mathrm{~mm}(\mathrm{~L}) \times 40 \mathrm{~mm}(\mathrm{~W}) \times 0.2$ $\mathrm{mm}(\mathrm{T})$ ), made of Kapton ${ }^{\oplus}$ polyimide film and tape (Figure S10), and pressing $(10 \mathrm{MPa})$ at $120^{\circ} \mathrm{C}$ for 5 min. Broken and cut pieces of PSN76 films were gathered and reprocessed to the original form using the same procedure.

\section{Instrumentation and Characterization Techniques}

The sulfur content of PSNs was analyzed using an elemental analyzer (EA, Flash 2000, Thermo Scientific). Scanning electron microscope with energy dispersive X-ray spectroscopy (SEM-EDS, $\mathrm{XFlash}^{\oplus}$ Detector 5030) was used to confirm the residual $\mathrm{I}_{2}$ in the PSNs. Fourier transform infrared (FT-IR) spectra were recorded on an Bruker Alpha II FTIR spectrometer using attenuated total reflectance (ATR) equipment. The thermal stability of PSNs was investigated by thermal gravimetric analysis (TGA) using a TA Instruments TGA Q5000 under a nitrogen atmosphere. The samples were heated from room temperature to $800{ }^{\circ} \mathrm{C}$ at a heating rate of $10{ }^{\circ} \mathrm{C} / \mathrm{min}$. Differential scanning calorimetry (DSC) was run using a TA Instruments DSC Q2000 under a nitrogen atmosphere. Samples with a typical mass of 5-10 mg were encapsulated in sealed aluminum pans. They were first heated from $25^{\circ} \mathrm{C}$ to $150^{\circ} \mathrm{C}$ and then cooled down to $0{ }^{\circ} \mathrm{C}$, which were followed by second heating to $150{ }^{\circ} \mathrm{C}$ at a constant rate of $10^{\circ} \mathrm{C} \mathrm{min}-1$. Wide-angle X-ray diffraction (WAXD) was performed using a Rigaku SmartLab. The applied voltage and current were $40 \mathrm{kV}$ and $40 \mathrm{~mA}$, respectively. The samples were mounted on aluminum holder and scanned from $2^{\circ}$ to $60^{\circ}$. Solvent extraction experiment was performed by placing a small piece (ca. $20 \mathrm{mg}$ ) of PSNs films into a 20 $\mathrm{mL}$ vial filled with toluene. After stored in an oven at $25^{\circ} \mathrm{C}$ for $24 \mathrm{~h}$, the film was recovered and dried at $70{ }^{\circ} \mathrm{C}$ under vacuum for $18 \mathrm{~h}$. Gel fraction $\left(f_{\mathrm{g}}\right)$ was calculated as

$$
f_{\mathrm{g}}=W_{\mathrm{a}} / W_{\mathrm{d}}
$$

where $W_{\mathrm{d}}$ and $W_{\mathrm{a}}$ are the weights of dried film before and after the toluene solvent extraction. Dynamic mechanical analysis (DMA) was performed on a TA Instruments DMA Q800 with attached cryo accessory using rectangular-shaped PSNs films (ca. $20 \mathrm{~mm}(\mathrm{~L}) \times 5 \mathrm{~mm}(\mathrm{~W}) \times 0.2 \mathrm{~mm}$ (T)). DMA temperature sweep experiment was conducted in the film tension mode with a $1 \mathrm{~Hz}$ frequency, $0.1 \%$ strain, and $0.1 \mathrm{~N}$ axial force. The specimens were first cooled down from room 
temperature to $-100{ }^{\circ} \mathrm{C}$ and then heated to $100{ }^{\circ} \mathrm{C}$ at a constant rate of $5{ }^{\circ} \mathrm{C} \mathrm{min}{ }^{-1}$ in a nitrogen atmosphere. Cross-linking density $\left(v_{\mathrm{e}}\right)$ of PSNs was calculated as

$$
v_{\mathrm{e}}=E^{\prime} / 3 R T
$$

where $E^{\prime}, R$, and $T$ are the storage modulus, universal gas constant, and absolute temperature in the rubbery region, respectively. The temperature-dependent rheological properties of PSNs were characterized using a rotational rheometer (ARES-G2, TA Instruments) with a $0.1 \%$ strain, $1 \mathrm{~Hz}$ frequency, and constant rate of $5{ }^{\circ} \mathrm{C} \mathrm{min}^{-1}$ to confirm the rubbery plateau before the polymer degradation due to the thermally induced S-S bond cleavages. Frequency sweep experiment was also conducted on the same rheometer with a $0.1 \%$ strain at different temperatures above $T_{g} s$ of the PSNs. The specimens were first equilibrated at each temperature and the frequency was swept from 0.1 to $100 \mathrm{rad} \mathrm{s}^{-1}$. Uniaxial tensile testing was carried out on an Instron LR5K universal testing machine (UTM, Lloyd Instruments) at a strain rate of $0.0083 \mathrm{~s}^{-1}\left(10 \mathrm{~mm} \mathrm{~min}^{-1}\right)$. Rectangular shaped tensile bars (ca. $40 \mathrm{~mm}(\mathrm{~L}) \times 5 \mathrm{~mm}(\mathrm{~W}) \times 0.2 \mathrm{~mm}(\mathrm{~T})$, gauge length $=20 \mathrm{~mm}$ ) were stamped out from the films using a cutting die. At least three different samples were prepared from each film and tested in the UTM. Cyclic tensile testing was performed on the same UTM using the rectangular shaped PSN76 films at a strain rate of $0.0083 \mathrm{~s}^{-1}\left(10 \mathrm{~mm} \mathrm{~min}^{-1}\right)$. All the tensile tests were performed at room temperature and humidity $\left(25 \pm 1{ }^{\circ} \mathrm{C}, \mathrm{RH} 45 \pm 8 \%\right.$ in air). Refractive index measurements were conducted on a Metricon 2010/M prism coupler using $200 \mu \mathrm{m}$-thick PSN46, 63, and 76 films. Refractive indices which are parallel $\left(n_{\|}\right)$and perpendicular $\left(n_{\perp}\right)$ to the film plane were measured as a function of wavelength. To investigate the UV- and heat-induced scratch-healing behaviors of PSN76 film, a UV LED (365 nm, $2 \mathrm{~W} \mathrm{~cm}^{-2}$, FE400, Phoseon Technology) and a temperature-controlled vacuum oven (OV-12, Jeio Tech) were used, respectively. The scratch depths and healing behaviors on the PSN76 film were observed using an alpha-step surface profiler ( $\alpha$-step DC50, KLA-Tencor) and a Nikon Eclipse microscope (50i POL), respectively. An infrared (IR) camera (T300, FLIR) was utilized to capture ambient temperature long-IR $(7.5-13 \mu \mathrm{m})$ thermograms. 


\section{Synthesis of Poly(phenylene polysulfide) Networks (PSNs)}

\section{a Process $\mathrm{A}$}

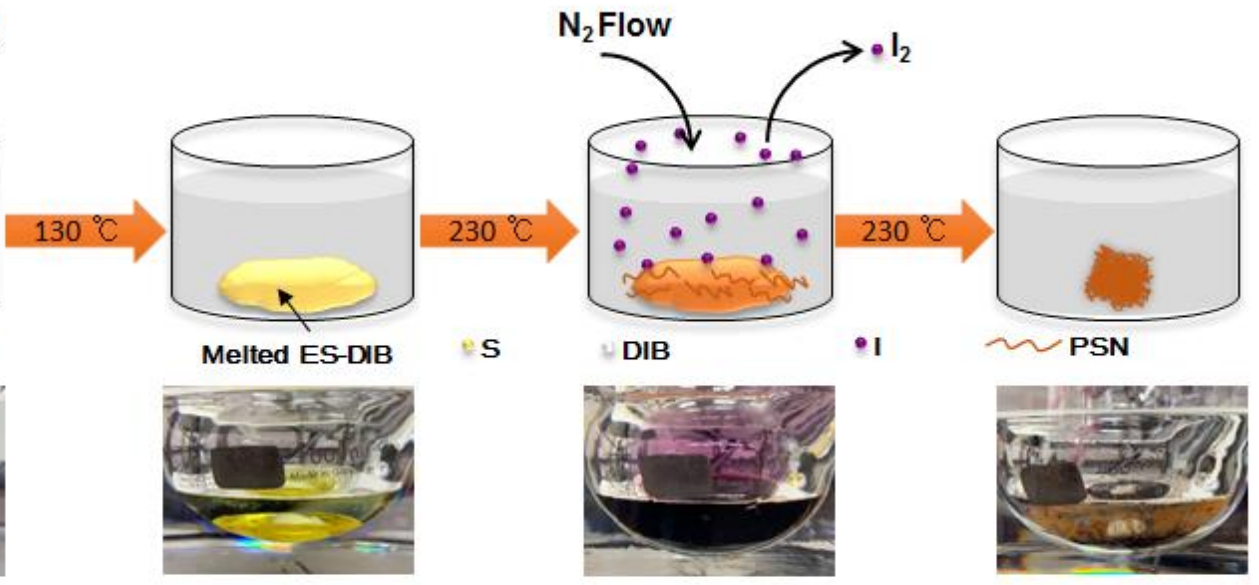

\section{b Process B}
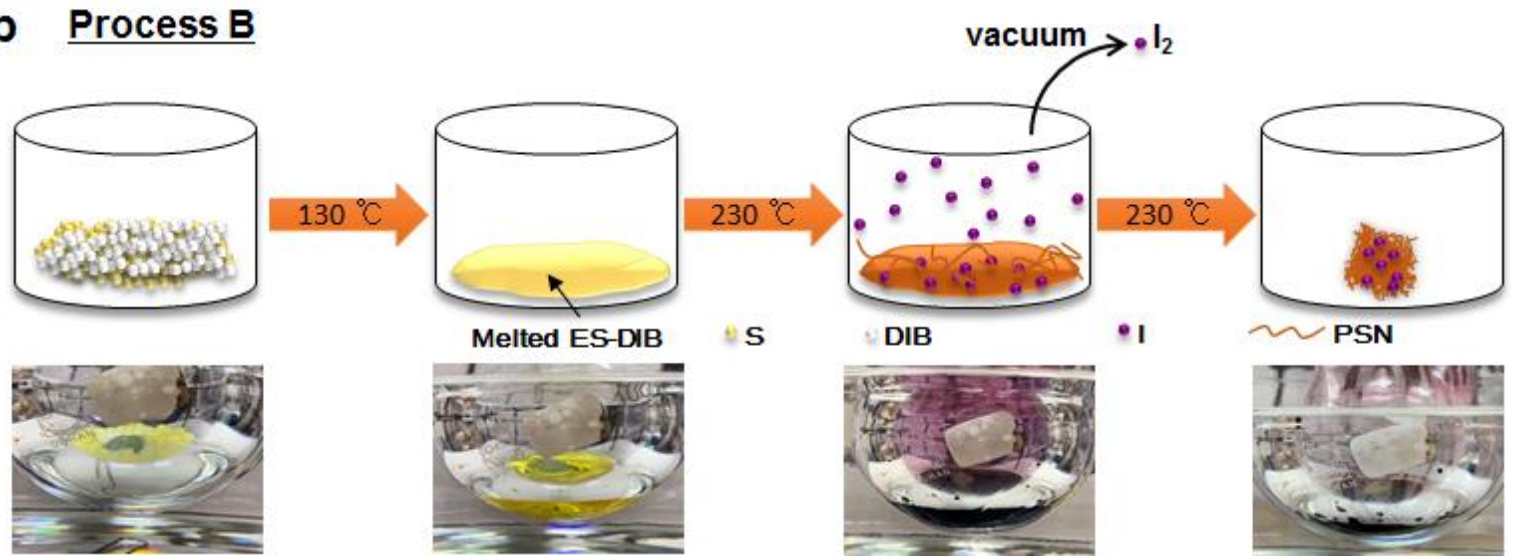

Figure S1. Schematic illustrations and corresponding photographs of the polymerization processes: (a) Polymerization with silicone oil as a reaction medium and $\mathrm{N}_{2}$ flow (Process $\mathrm{A}$ ) and (b) melt polymerization under vacuum ${ }^{\mathrm{s}, s 2}$ (Process $\mathrm{B}$ ). In comparison to the highly viscous PSN melts in the Process B, the PSNs in silicone oil (Process A) were readily stirred and fragmented into smaller droplets by a vigorous magnetic stirring, although the PSNs were distinctly phase-separated with the silicone oil. Such enhanced flowability and fragmentation of the PSN droplets in silicone oil could facilitate the evaporation and removal of generated $\mathrm{I}_{2}$ from the PSNs. 


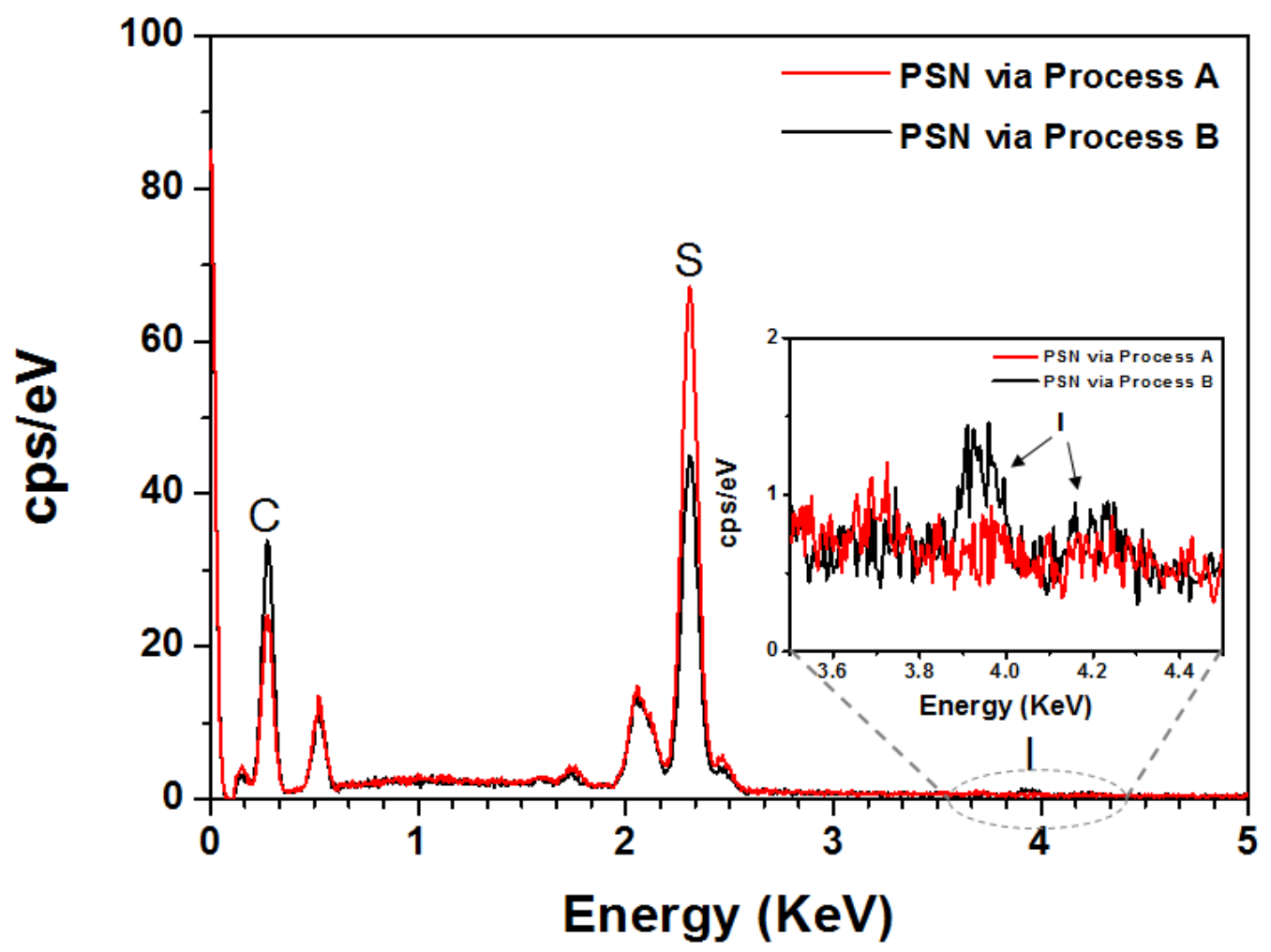

Figure S2. SEM-EDS analysis for PSNs (from the molar feed ratio of [ES]:[DIB] = 7:1) prepared via Process A (with acetone washing and drying to remove silicone oil) and Process B (without soxhlet extraction) in Figure S1. The smaller S/C ratio of the PSN prepared via Process B compared to that via Process A could reflect the larger loss of ES than DIB during the polymerization process under vacuum. Moreover, in contrast to that prepared via Process A, the PSN prepared via Process B shows $\mathrm{I}_{2}$ peaks present in the polymer matrix, thus additional purification process is required to remove the residual $\mathrm{I}_{2}$. 


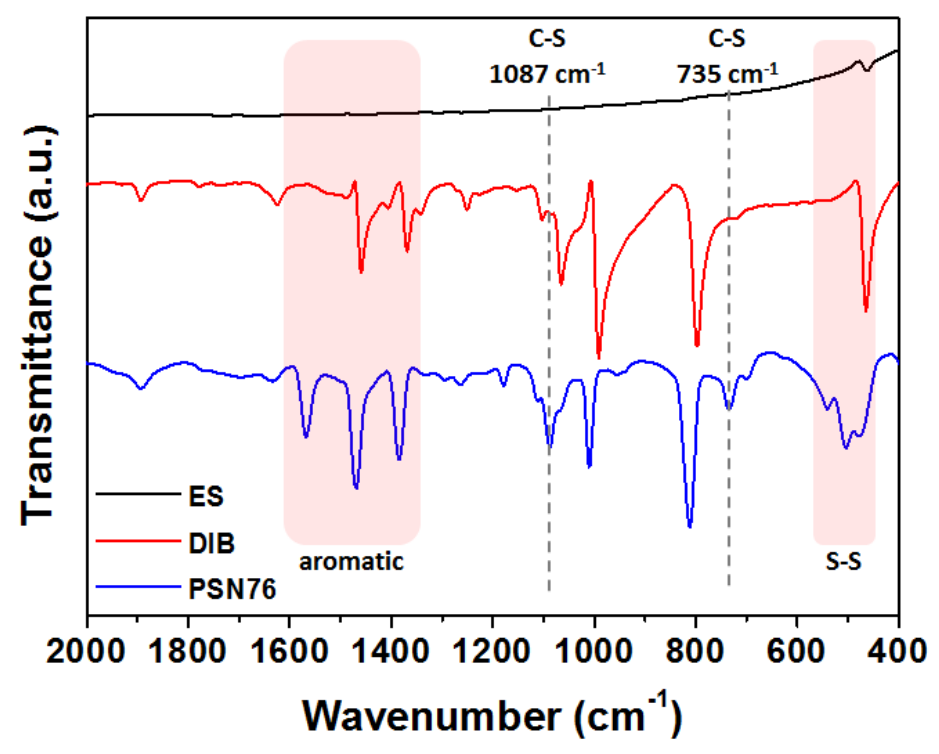

Figure S3. FT-IR spectra of ES, DIB, and PSN76.
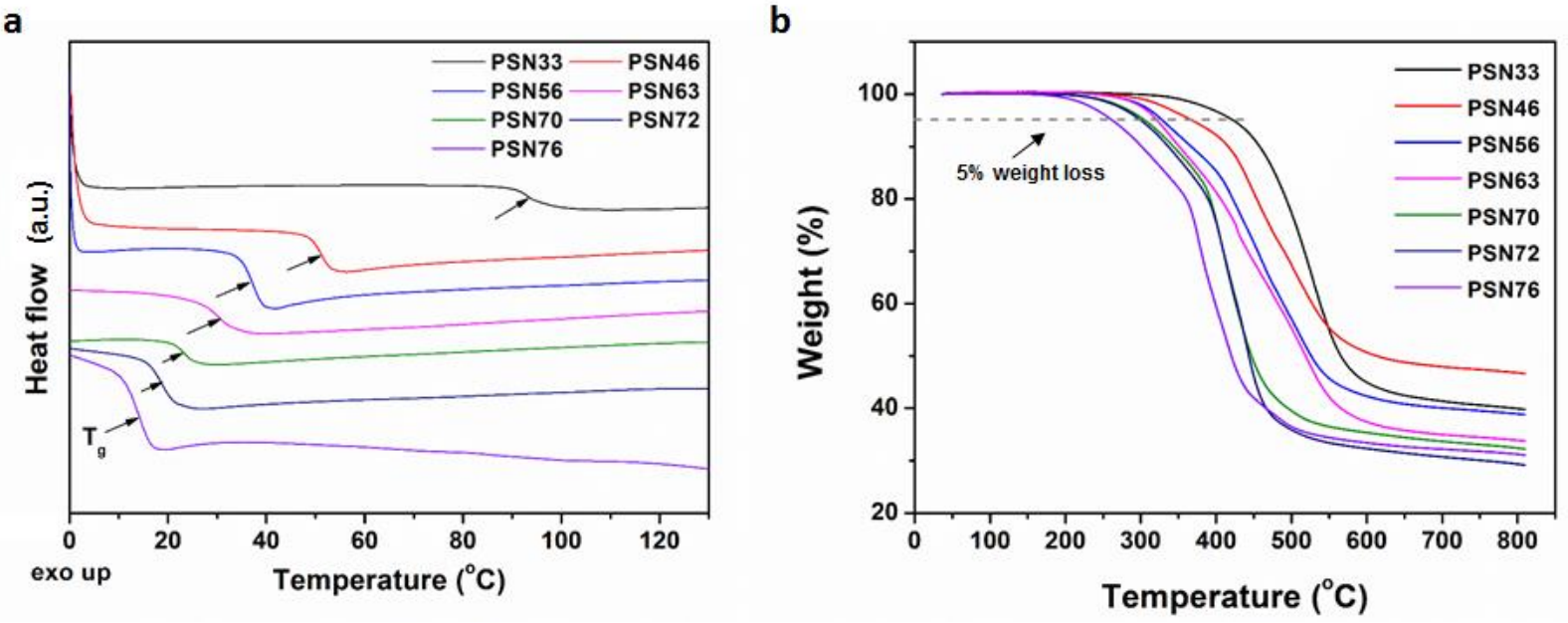

Figure S4. (a) DSC thermograms and (b) TGA curves of PSNs. 


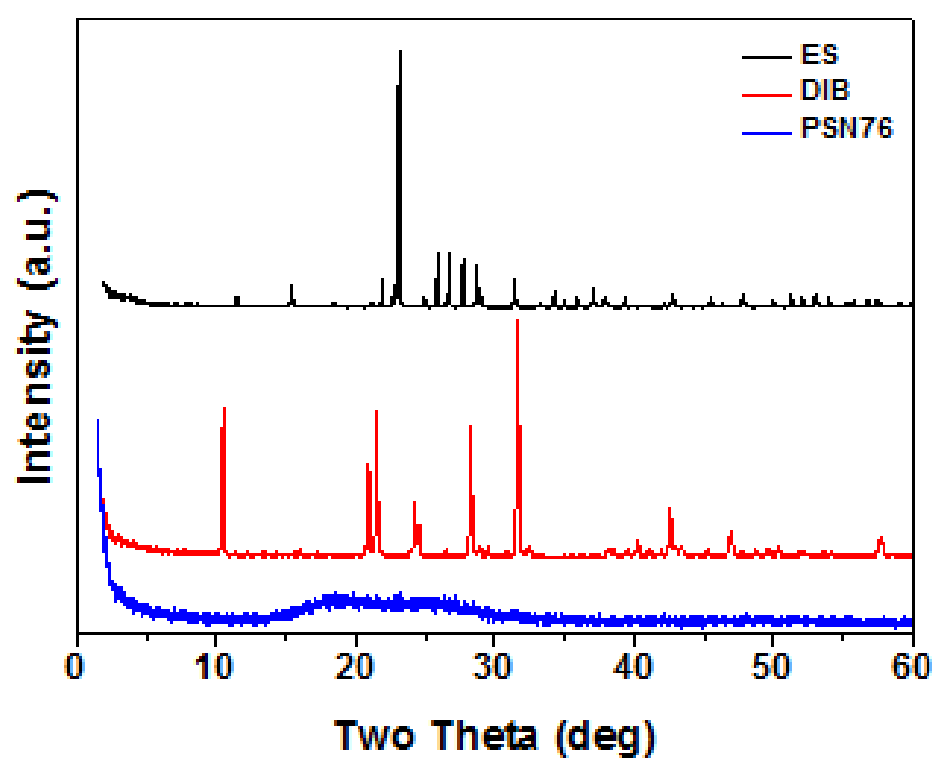

Figure S5. WAXD profiles of ES, DIB, and PSN76.

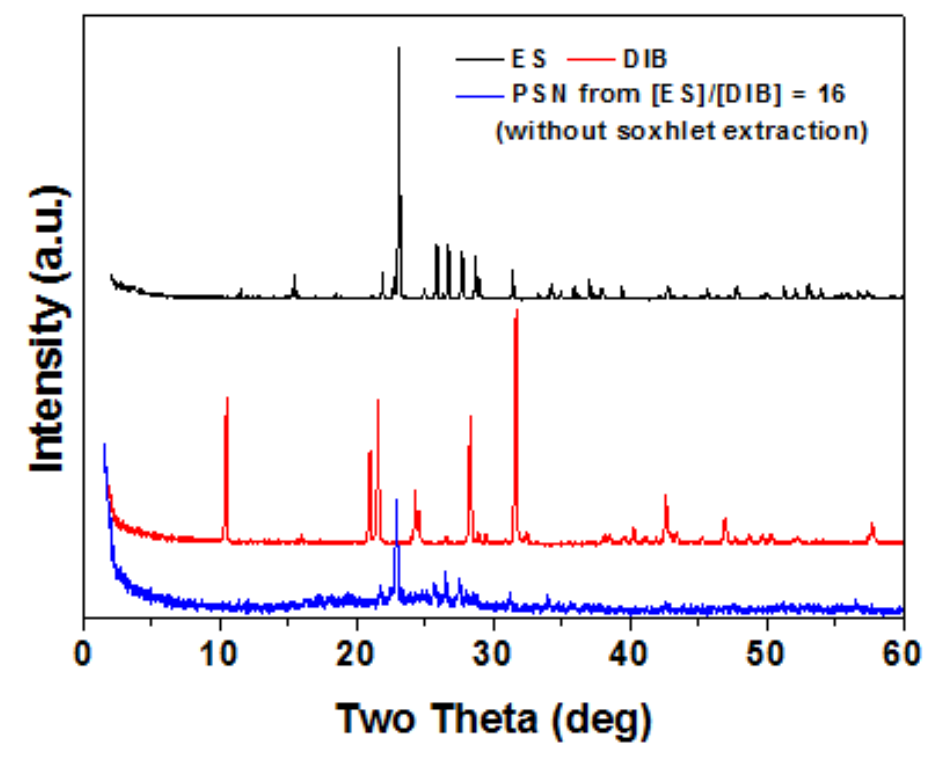

Figure S6. WAXD profiles of ES, DIB, and a PSN (without soxhlet extraction) prepared from a molar ES to DIB feed ratio ([ES]/[DIB]) of 16 . 


\section{Structural Analysis of PSNs}

Table S1. Gel Fraction and Cross-linking Density of PSN46, PSN63, and PSN76.

\begin{tabular}{cccc}
\hline & PSN46 & PSN63 & PSN76 \\
\hline $\boldsymbol{f}_{\mathrm{g}}^{a}$ & 0.97 & 0.96 & 0.98 \\
$\boldsymbol{v}_{\mathrm{e}}{ }^{b}\left(\mathrm{~mol} / \mathbf{m}^{3}\right)$ & 824 & 261 & 181 \\
\hline
\end{tabular}

${ }^{a}$ Gel fraction, obtained by $f_{\mathrm{g}}=W_{\mathrm{a}} / W_{\mathrm{d}}$, where $W_{\mathrm{d}}$ and $W_{\mathrm{a}}$ are the weights of dried film before and after toluene solvent extraction. ${ }^{b}$ Cross-linking density, calculated by $v_{\mathrm{e}}=E^{\prime} / 3 R T$, where $E^{\prime}, R$, and $T$ are the storage modulus, universal gas constant, and absolute temperature in the rubbery region, respectively. $E^{\prime}$ values at 343, 338, and 333 K in Figure S7 were used for PSN46, PSN63, and PSN76, respectively.

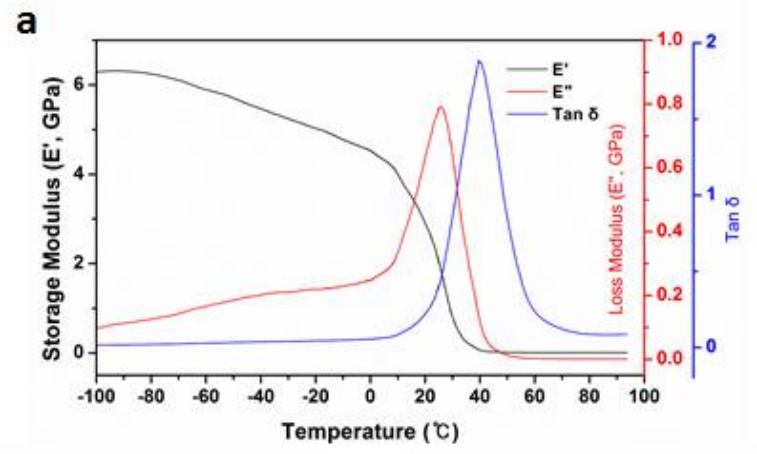

b

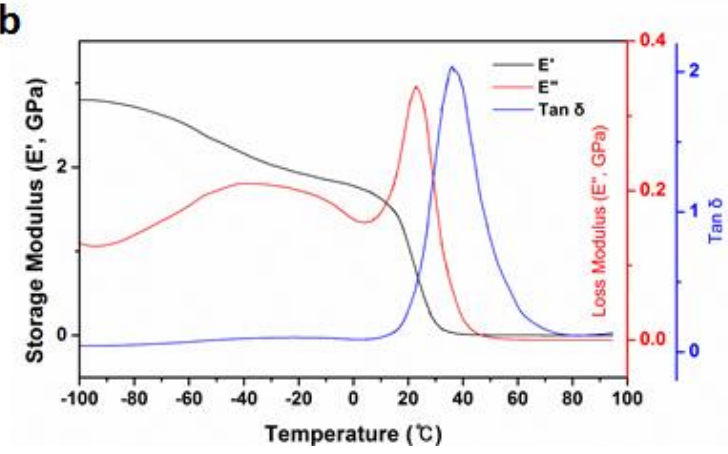

C

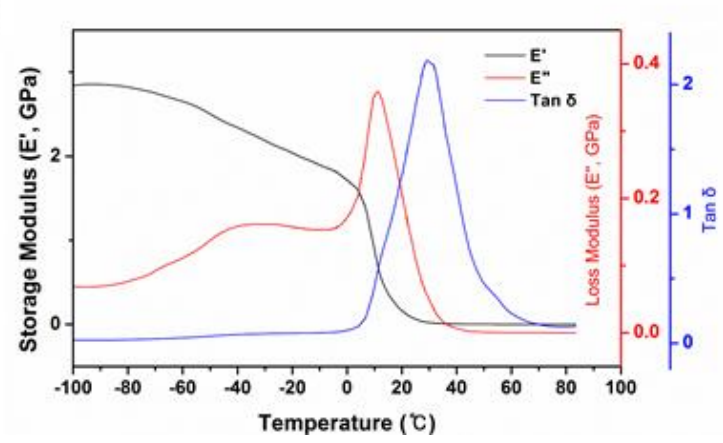

Figure S7. DMA curves of (a) PSN46, (b) PSN63, and (c) PSN76 films. 
a

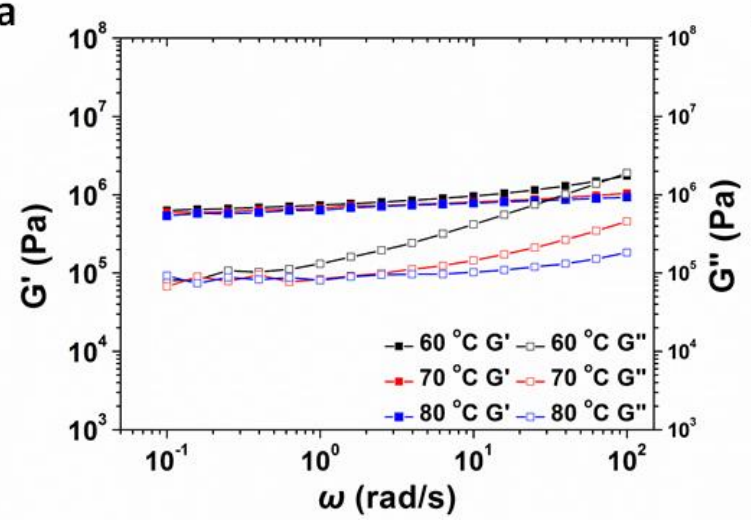

b

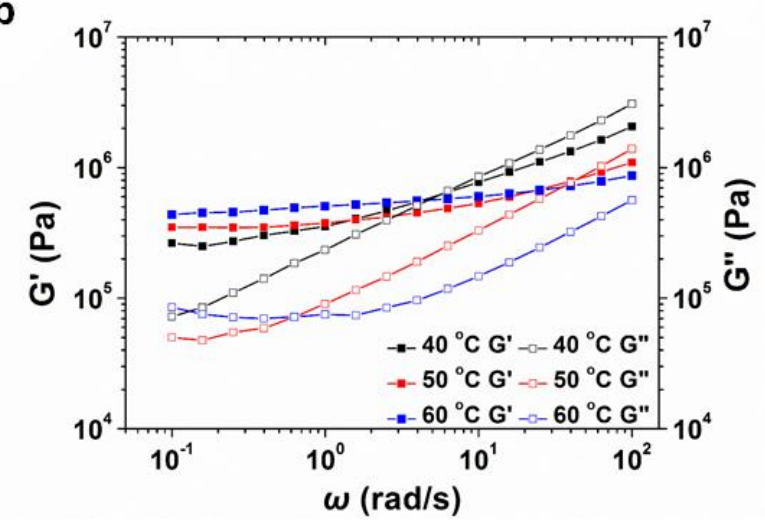

Figure S8. Frequency sweeps performed for (a) PSN46 and (b) PSN63 films at various temperatures slightly higher than $T_{\mathrm{g}}$ s. 


\section{Mechanical Characterization of PSN Films}

Table S2. Summary of mechanical properties of PSN46, PSN63, and PSN76 films.

\begin{tabular}{ccccc}
\hline & PSN46 & PSN63 & PSN76 & r-PSN76 $^{b}$ \\
\hline $\begin{array}{c}\boldsymbol{E}^{a} \\
(\mathbf{M P a})\end{array}$ & $37 \pm 5$ & $29 \pm 15$ & $7 \pm 2$ & $12 \pm 5$ \\
$\begin{array}{c}\boldsymbol{\sigma}_{\mathbf{u}}{ }^{a} \\
(\mathbf{M P a})\end{array}$ & $1.7 \pm 0.1$ & $1.6 \pm 0.1$ & $0.9 \pm 0.1$ & $1.0 \pm 0.1$ \\
$\boldsymbol{\varepsilon}_{\mathbf{b}}{ }^{a}$ & $170 \pm 10$ & $240 \pm 10$ & $300 \pm 10$ & $290 \pm 20$ \\
$(\%)$ & & & & \\
\hline
\end{tabular}

${ }^{a}$ Determined from tensile testing at room temperature $\left(25 \pm 1^{\circ} \mathrm{C}\right)$, where $E, \sigma_{\mathfrak{u}}$, and $\varepsilon_{\mathfrak{b}}$ are elastic modulus, ultimate tensile stress, and strain at break, respectively. ${ }^{b}$ Reprocessed from broken PSN76 film under tensile stress.

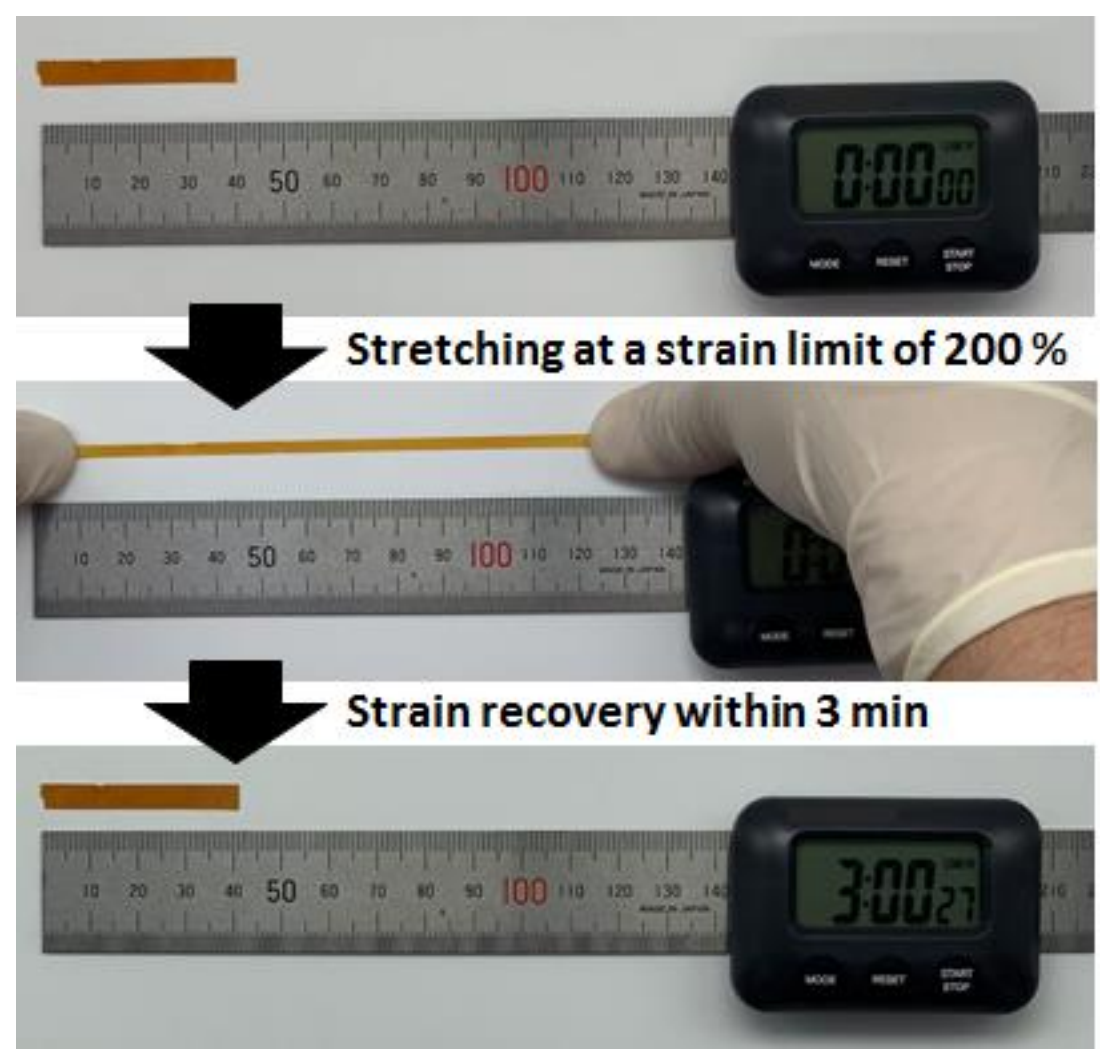

Figure S9. Photographs of strain recovery behavior of PSN76 film after stretching at a strain limit of $200 \%$. 


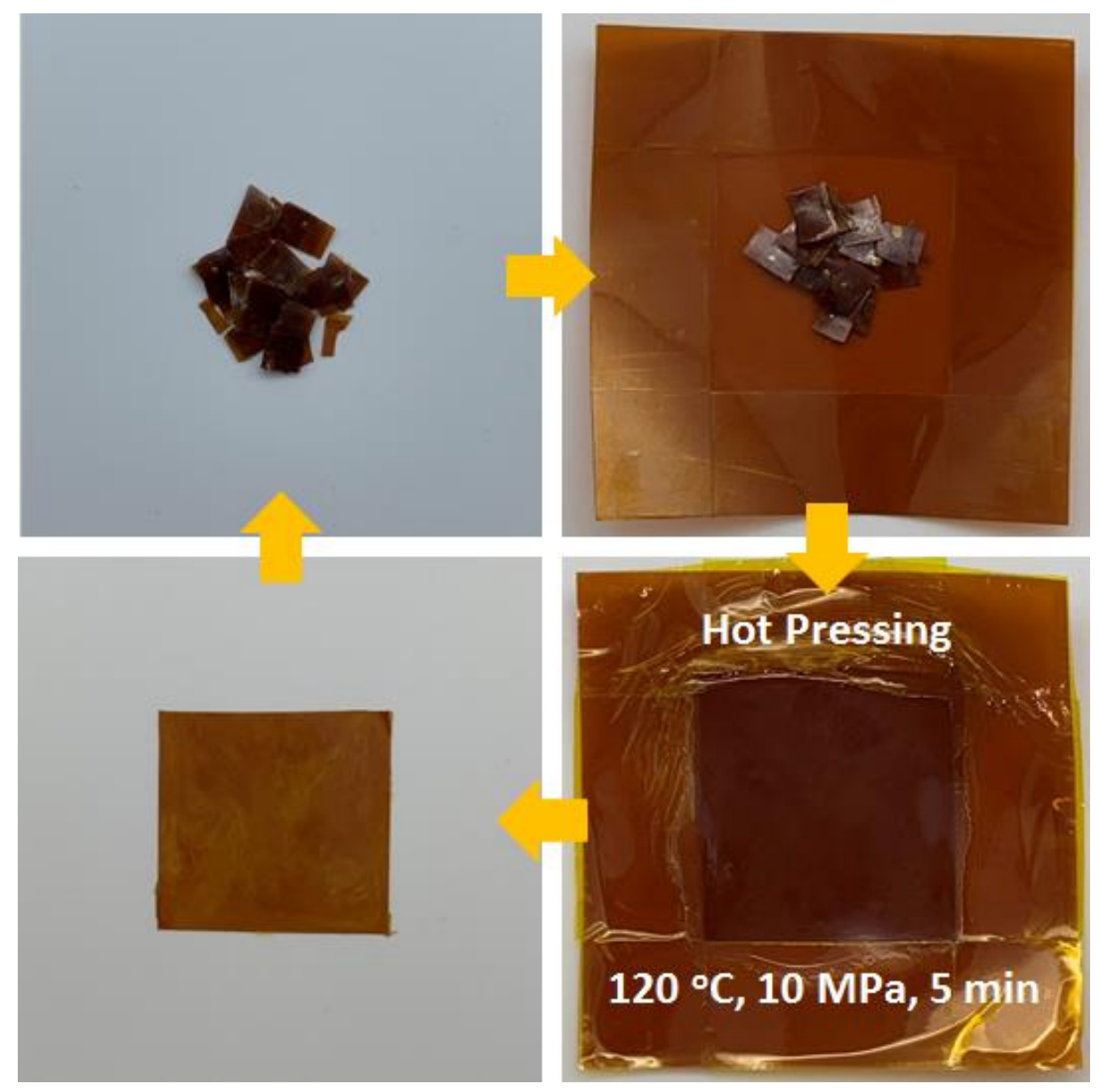

Figure S10. Photographs of reprocessing of PSN76 film after breaking under tensile stress and cutting into small pieces. 


\section{Optical Properties of PSN Films}

Table S3. Refractive indices $\left(n_{\|}, n_{\perp}\right.$, and $\left.n_{\text {av }}\right)$ of PSN46, PSN63, and PSN76 films at different wavelengths.

\begin{tabular}{ccccccccccc}
\hline \multirow{2}{*}{$\begin{array}{c}\text { Wavelength } \\
(\mathbf{n m})\end{array}$} & \multicolumn{3}{c}{ PSN46 } & \multicolumn{3}{c}{ PSN63 } & \multicolumn{3}{c}{ PSN76 } \\
\cline { 2 - 9 } & $n_{\|}$ & $n_{\perp}$ & $n_{\mathrm{av}}{ }^{a}$ & $n_{\|}$ & $n_{\perp}$ & $n_{\mathrm{av}}{ }^{a}$ & $n_{\|}$ & $n_{\perp}$ & $n_{\mathrm{av}}{ }^{a}$ \\
\hline $\mathbf{5 8 9}$ & 1.834 & 1.799 & 1.817 & 1.894 & 1.869 & 1.882 & 1.936 & 1.911 & 1.924 \\
$\mathbf{6 3 3}$ & 1.810 & 1.789 & 1.800 & 1.873 & 1.854 & 1.864 & 1.913 & 1.896 & 1.905 \\
$\mathbf{7 3 0}$ & 1.783 & 1.777 & 1.780 & 1.851 & 1.839 & 1.845 & 1.885 & 1.875 & 1.880 \\
$\mathbf{8 1 6}$ & 1.776 & 1.772 & 1.774 & 1.843 & 1.837 & 1.840 & 1.876 & 1.865 & 1.871 \\
\hline
\end{tabular}

${ }^{a}$ Averaged refractive index value of $n_{\|}$and $n_{\perp}$.

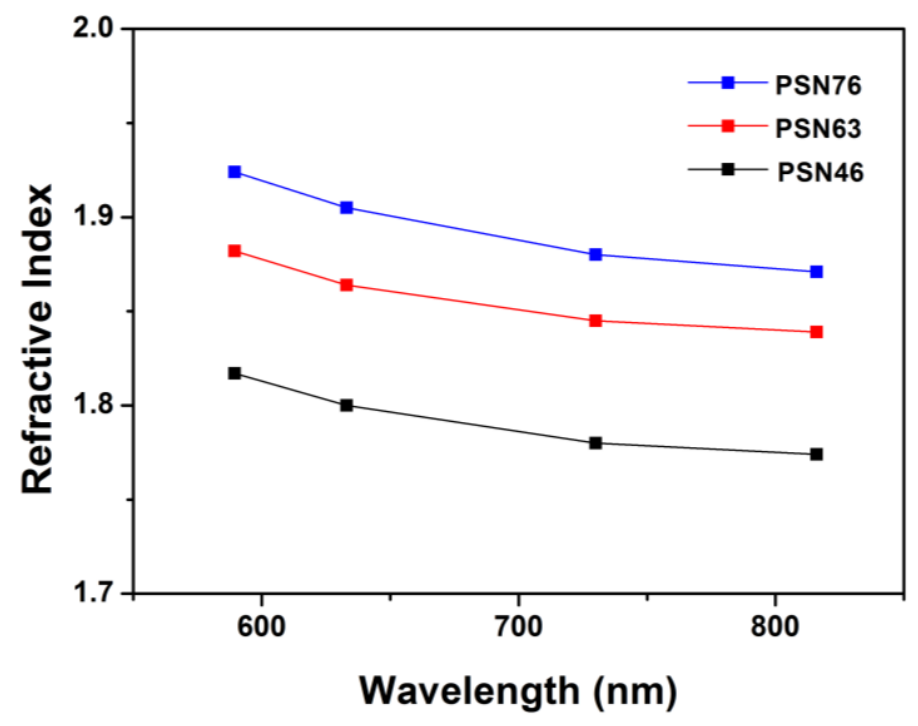

Figure S1 1. Plots of average refractive index $\left(n_{\mathrm{av}}\right)$ vs. wavelength for $200 \mu \mathrm{m}$-thick PSN46, PSN63, and PSN76 films. 


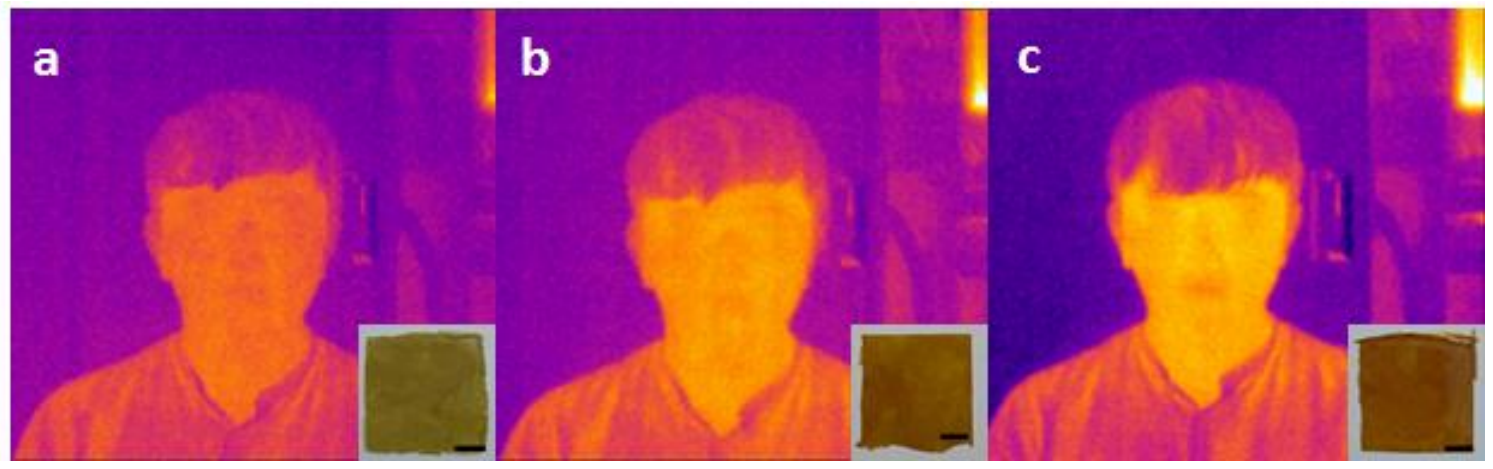

Figure S12. Thermal images of a male subject captured through (a) PSN46, (b) PSN63, and (c) PSN76 films (scale bar $=1 \mathrm{~cm})$.

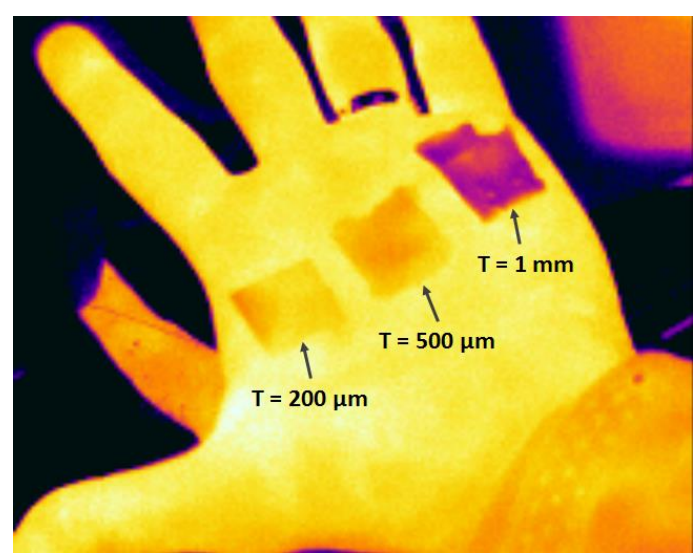

Figure S13. An IR thermal image of PSN76 films with different thickness on a hand subject.

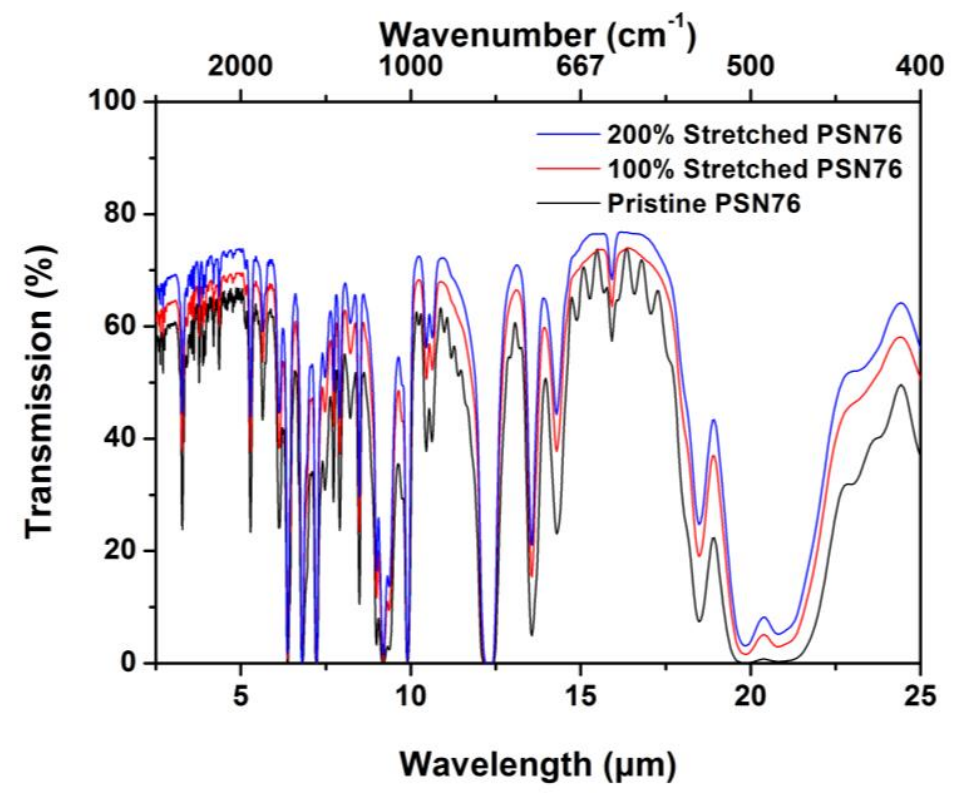

Figure S14. FT-IR transmission spectra of PSN76 film (pristine film dimension: ca. $5 \mathrm{~mm}(\mathrm{~L}) \times 20$ $\mathrm{mm}(\mathrm{W}) \times 200 \mu \mathrm{m}(\mathrm{T}))$ with different strain values. 

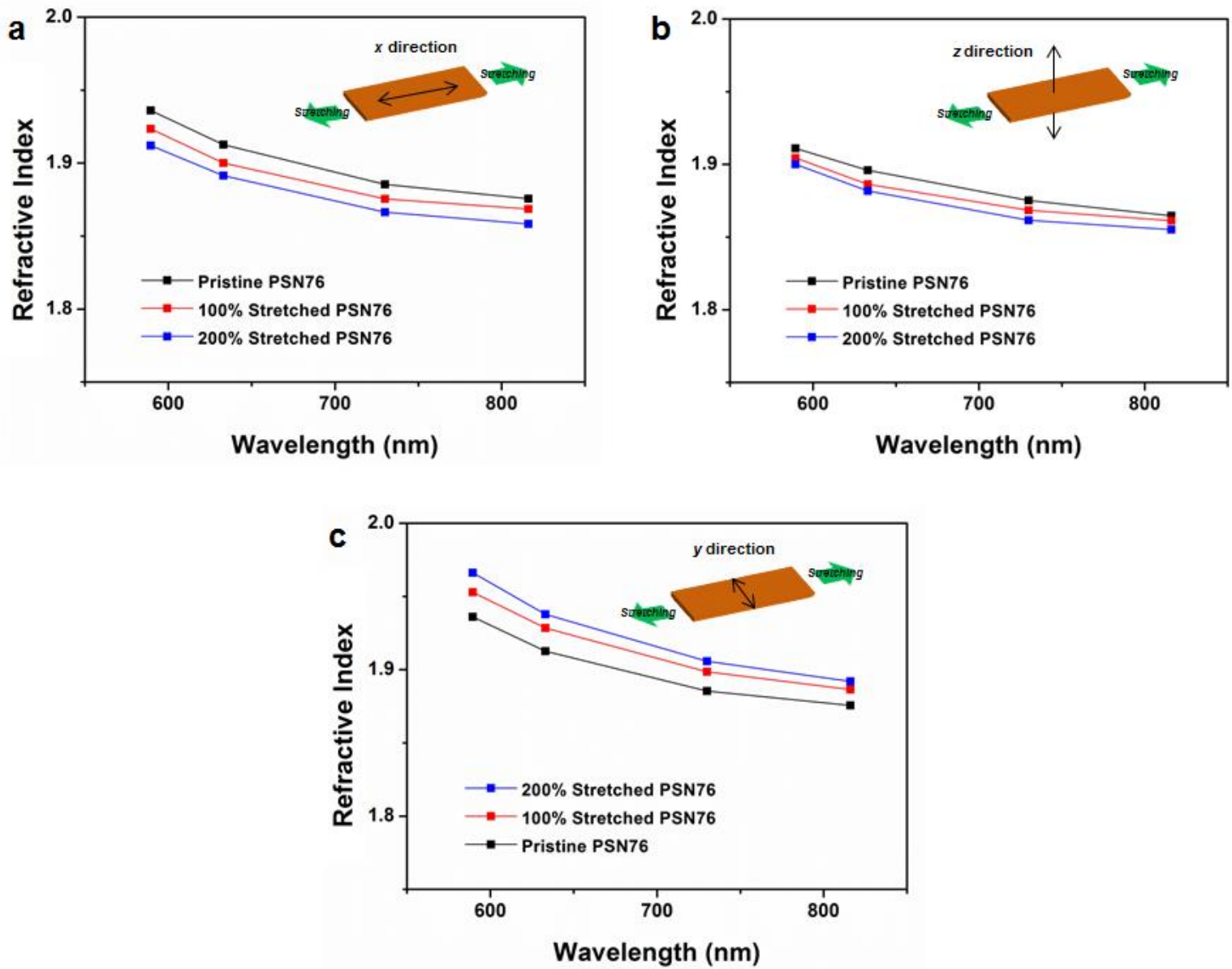

Figure S15. Refractive indices of PSN76 film (pristine film dimension: ca. $5 \mathrm{~mm}(\mathrm{~L}) \times 20 \mathrm{~mm}(\mathrm{~W})$ $\times 200 \mu \mathrm{m}(\mathrm{T}))$ with different strain values, measured in the (a) $x$ direction parallel to the stretching direction on the film plane, (b) $z$ direction perpendicular to the film plane, and (c) $y$ direction perpendicular to the stretching direction on the film plane. 


\section{References for Supporting Information}

(S1) Rule, M.; Fagerburg, D. R; Watkins, J. J.; Lawrence, P. B. A New Melt Preparation Method for Poly(phenylene sulfide). Makromol. Chem., Rapid Commun. 1991, 12, 221-226.

(S2) Rule, M.; Fagerburg, D. R.; Watkins, J. J.; Lawrence, P. B.; Zimmerman, R. L.; Cloyd, J. D. Some Characteristics of a New and Novel Melt Preparation Method for Poly(phenylene sulfide). Makromol. Chem., Macromol. Symp. 1992, 54/55, 233-246. 